\title{
Research on the Inter-harmonics Equivalent Impedance of Series Hybrid Active Power Filter
}

\author{
Zhang Jian-gong*, Liu Jian-ben ${ }^{\dagger}$, Dai Shao-jun**, Chen Qiao-fu* and He Jun-jia*
}

\begin{abstract}
In the series hybrid active power filter (SHAPF) with magnetic flux compensation (MFC), the system current oscillate in the experimental results when adding the same phase harmonic current command in current control block. This condition endangers the security of the SHAPF. Taking the digit period average arithmetic as example, this paper explains the inter-harmonics current oscillation in the experiment. The conclusion is that the SHAPF is unstable to the inter-harmonics current in theory. Limited by the capacity of the inverter, the system current and the inverter output current do not increase to infinite. At last, some methods are proposed to solve this problem. From the practical viewpoint, the voltage feed-forward control is easy to achieve. It can suppress the current oscillation problems, and also improve the filtering effect. The feasibility of the methods is validated by both the emulation and experiment results.
\end{abstract}

Keywords: Active power filter, Series transformer, Inter-harmonics oscillation, Voltage feed-forward control

\section{Introduction}

As the wide application of power electronic equipments and nonlinear loads, the harmonic propagation in power distribution systems become a serious problem in the recent decades. In order to suppress harmonics, many methods have been proposed. Shunt passive power filters (PPF) have been widely used to keep harmonic contamination within acceptable limits. However, shunt PPF have many disadvantages in practical application [1-2]. They may cause resonance and the impedance of the power system strongly affects the filtering effects. To overcome these disadvantages, Active Power Filter (APF) has been proposed [3-8]. A novel principle of magnetic flux compensation (MFC) is proposed in reference [7], which has been applied in series hybrid APF [7-8], controllable reactor [9], and shunt hybrid APF [10]. In the series hybrid APF, the series transformer works as a controlled reactor. The series transformer exhibits primary leakage impedance to the fundamental current and magnetizing impedance to the harmonic current with the proper control of pulse-width modulation (PWM) inverters, then the harmonic currents are forced to flow into the passive filter.

Since the series hybrid APF requires the series transformer exhibits low impedance to the fundamental current and high impedance to the harmonic current, a low

$\dagger$ Corresponding Author: Hubei Key Laboratory of Power Network Environmental Protection, China Electric Power Research Institute, Wuhan, China. (laolace@163.com)

* State Key Laboratory of Advanced Electromagnetic Engineering and Technology, Huazhong University of Science and Technology, China. / China Electric Power Research Institute, Wuhan, China.

** State Grid Wuhan Power Supply Company, Wuhan, China.

Received: November 27, 2013; Accepted: May 14, 2015 pass filter (LPF) is needed in the current control block to separate the fundamental current and harmonic current from the system current $I_{1}$. The properties of the actual LPF algorithm cannot be ideal. Some specific frequency inter-harmonics (Non-integer harmonics) may pass through the LPF and affect the inverter output current. As a result, the series transformer equivalent impedance to interharmonics is not equal to the equivalent impedance to harmonics. But in reference [7-8] and [11], this difference was ignored. All of them do not consider the properties of the LPF. They just simply separate the actual APF circuit to the fundamental equivalent circuit and harmonic (including inter-harmonics) equivalent circuit. In each equivalent circuit, the reference current $I_{\text {ref }}$ is proportional to the primary winding current of series transformer $I_{1}$. The equivalent impedance of the series transformer to the inter-harmonics has not been discussed independently. For this reason, the theoretical analysis and conclusions in reference [7-8] and [11] about the equivalent impedance of transformer are incorrect. In experimental, inter-harmonics oscillation phenomenon occurred when compensate both fundamental and harmonics current. The traditional analytical method cannot explain this inter-harmonics oscillation phenomenon.

This article firstly introduce in basic filtering principle of the series hybrid APF based on MFC, then explain the reason of the inter-harmonics oscillation phenomenon when compensating both fundamental and harmonics current by the inverter. The essence of the inter-harmonics oscillation is that the APF system is unstable to the inter-harmonics. Finally, voltage feed-forward control method is proposed to suppress the inter-harmonics oscillation. 


\section{Working Principle}

Fig. 1 shows the T-type equivalent circuit of a single phase series transformer. Assume all parameters in Fig. 1 are the equivalent value after winding convert. $r_{1 \sigma}, L_{1 \sigma}$, and $r_{2 \sigma}, L_{2 \sigma}$ represent the leakage impedance of the primary and secondary winding of the transformer; $r_{\mathrm{m}}$ and $L_{\mathrm{m}}$ represent the magnetizing impedance; $i_{1}$ is the current in the primary winding; $i_{2}$, which is generated by a PWM inverter, is also the current in the secondary winding. It contains $-\alpha$ times of the fundamental current and $\beta$ times of the harmonic current of $I_{1}$. That is to say:

$$
I_{2}=-\alpha \times I_{1}^{(1)}+\beta \times \sum_{\mathrm{n}=2}^{\infty} I_{1}^{(\mathrm{n})}
$$

The leakage inductance can be ignored since $L_{\mathrm{m}}>>$ $L_{1 \sigma}+L_{2 \sigma}$. The resistance of the series transformer can also be ignored since they are very small. Hence the voltage equation of the transformer primary winding is:

$$
\left\{\begin{array}{l}
U_{1}^{(1)} \approx I_{1}^{(1)} \omega(1-\alpha) L_{\mathrm{m}} \\
U_{1}^{(\mathrm{n})} \approx I_{1}^{(\mathrm{n})} n \omega(1+\beta) L_{\mathrm{m}}
\end{array}\right.
$$

where: $\omega$ - the fundamental angular frequency

In Eq. (2), $0<\alpha \leq 1, \beta>0$. If $\alpha$ is equal to 1 , the series transformer will established nearly zero impedance to the fundamental, and $(1+\beta)$ times magnetizing impedance to the harmonics. The primary winding of the series transformer is inserted in series connection between the power grid and passive filter. Then the harmonic current are forced to inflow into the passive filter.

The detection block of the reference signals as Fig. 2 shows [12]. $\sin \omega t$ and $\cos \omega t$ are the $50 \mathrm{~Hz}$ sinusoidal signal and cosine signal. $i$ is the current of the primary winding, it contains fundamental and harmonic component:

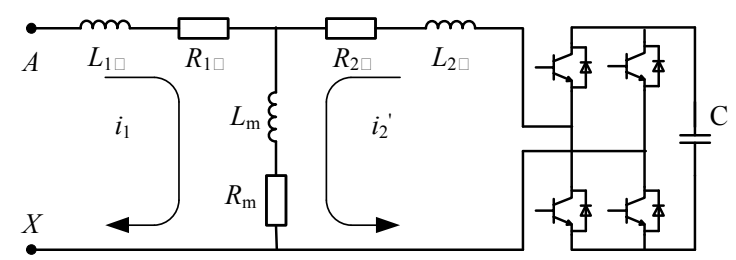

Fig. 1. Equivalent T-type circuit of series transformer

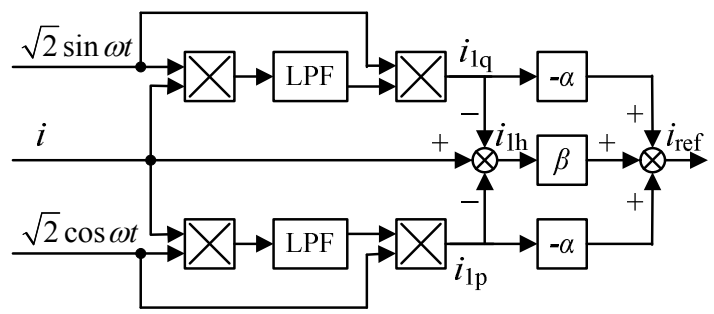

Fig. 2. Calculation of the reference signals

$$
i_{1}=\sqrt{2} \sum_{\mathrm{n}=1}^{\infty} I_{\mathrm{n}} \cos \left(n \omega t+\varphi_{\mathrm{n}}\right)
$$

Multiply $i_{1}$ by sinusoidal signal,

$$
\begin{aligned}
\sqrt{2} \sin (\omega t) \cdot i_{1}= & \sum_{\mathrm{n}=1}^{\infty} I_{\mathrm{n}}\left\{\sin \left[(n+1) \omega t+\varphi_{\mathrm{n}}\right]-\right. \\
& \left.\sin \left[(n-1) \omega t+\varphi_{\mathrm{n}}\right]\right\}
\end{aligned}
$$

If the LPF block has ideal low pass characteristic, only DC signal can through it:

$$
i_{1 \mathrm{q}}=-I_{1} \sin \varphi_{1}
$$

In a similar way, multiply $i_{1}$ by cosine signal, the other DC signal component can obtained:

$$
i_{1 \mathrm{p}}=I_{1} \cos \varphi_{1}
$$

The fundamental component of $i_{1}$ current is:

$$
\begin{aligned}
i_{1}^{(1)} & =\sqrt{2} \cos \omega t \cdot I_{1 \mathrm{p}}+\sqrt{2} \sin \omega t \cdot I_{1 \mathrm{q}} \\
& =\sqrt{2} I_{1} \cos \omega t
\end{aligned}
$$

The harmonic component can obtain as follow:

$$
i_{1 \mathrm{~h}}=i-i_{1}^{(1)}
$$

Finally, the reference current signals are:

$$
i_{\text {ref }}=-\alpha \times i_{1}^{(1)}+\beta \times i_{\text {lh }}
$$

\section{Inter-harmonic Oscillation}

\subsection{Oscillation phenomenon}

Inter-harmonics oscillation occurred when $\beta$ is not equal to 0 in the experiment. Fig. 3 shows the system current when the APF is under working condition $\alpha=1, \beta=1$. The system current waveform is "oscillating". Change the time coordinate to $50 \mathrm{~ms} / \mathrm{div}$, the system current voltage is shown as the $2^{\text {nd }}$ waveform in Fig. 3. The "beat" of the waveform indicates that there are inter-harmonics in the system current and the inter-harmonics frequency is close to Chinese power frequency---50Hz.

\subsection{Oscillation reason analysis}

Fig. 2 shows the reference current calculation algorithm. The fundamental and harmonic current detecting method has been explained in detail in [12]. By multiplying of 


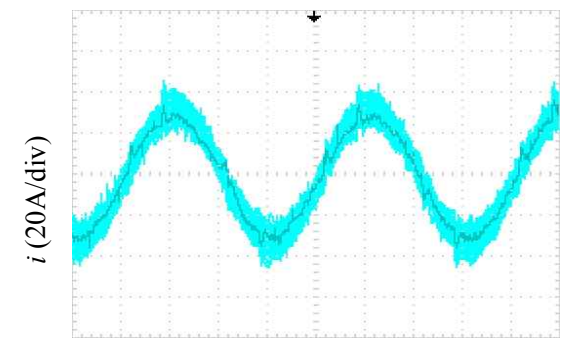

$t(5 \mathrm{~ms} / \mathrm{div})$

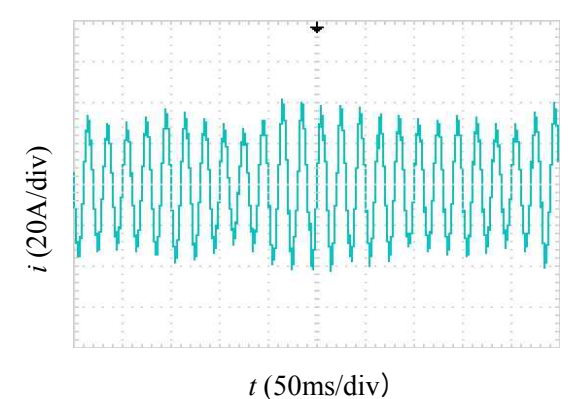

Fig. 3. The experiment system current waveform

sinusoidal and cosine signal, the $50 \mathrm{~Hz}$ fundamental current signal was converted to DC current signal. That means this multiplication in fact was a process of "frequency coordinate transformation". The frequency of fundamental signal in actual coordinate frame is $50 \mathrm{~Hz}$, and in fictitious synchronization coordinate frame (after multiplying of sinusoidal and cosine signal), the frequency of fundamental signal is $0 \mathrm{~Hz}$.

The accurately mathematical model of this "frequency coordinate transformation" is quite complex [13] and it is not necessary for the following theoretical analysis of this manuscript. For this reason, this manuscript does not considering the difference of the inter-harmonics frequency in different coordinate system. The amplitude-frequency and phase-frequency response of the LPF to DC signal in Fig. 4 represent the amplitude-frequency and phasefrequency response of the reference current signal calculation system to the $50 \mathrm{~Hz}$ fundamental current signal.

The LPF in Fig. 2 using digit period average algorithm: $\mathrm{Y}=\mathrm{X}\left(\mathrm{z}^{-1}+\mathrm{z}^{-2}+\mathrm{z}^{-3} \ldots+\mathrm{z}^{-\mathrm{N}}\right) / \mathrm{N}$, hence the discrete transfer function of the digit period average algorithm LPF is:

$$
H_{\mathrm{LPF}}(z)=\frac{\left(1-z^{-N}\right)}{N(z-1)}
$$

If $\alpha=1$, the discrete transfer function of the Fig. 2 is:

$$
H(z)=\frac{I_{\mathrm{ref}}}{I_{1}}=\beta-(1+\beta) \frac{\left(1-z^{-N}\right)}{N(z-1)}
$$

In the APF, sampling frequency is $12.8 \mathrm{kHz}$; the sampling number in one fundamental period is $\mathrm{N}=256$. The bode diagram of Eq. (5) is shown as Fig. 4. The abscissa of Fig. 4 is $f_{\mathrm{pq}}$, which is the frequency in fictitious synchronization coordinate system. If $\beta \neq 0$, to the interharmonics, $I_{\text {ref }}$ is not the same phase to the inter-harmonics of the system current. That means the series transformer does not establish $(1+\beta)$ times magnetizing impedance to the inter-harmonics.

Then discuss the equivalent inter-harmonics impedance of the series transformer. Considered the inverter as ideal controlled current source, then connect an ideal unit current source to the primary winding of the series transformer, the current of the secondary winding of the series transformer can be known from Fig. 4. The vector diagram of the series transformer is shown as Fig. 5. $\boldsymbol{I}_{1}$, which is the ideal unit current source, is also the current of the primary winding; $\boldsymbol{I}_{2}$ is the current of secondary winding. $\boldsymbol{U}_{\mathrm{m}}$ and $\boldsymbol{I}_{\mathrm{m}}$ are the magnetizing voltage and current. Since the leakage inductance has been ignored, $\boldsymbol{U}_{\mathrm{m}}$ is equal to the voltage of the primary winding. The equivalent impedance of the series transformer is:

$$
\begin{aligned}
Z_{\mathrm{t}} & =\frac{\dot{U}_{\mathrm{m}}}{\dot{I}_{\mathrm{m}}}=\frac{\left(\dot{I}_{1}+\dot{I}_{2}\right) \times \mathrm{j} \omega_{\mathrm{f}} L_{\mathrm{m}}}{\dot{I}_{1}}=\frac{\mathrm{j} \omega_{\mathrm{f}} L_{\mathrm{m}}\left(1 \angle 0+A_{\mathrm{f}} \angle \theta_{\mathrm{f}}\right)}{1 \angle 0} \\
& =-\omega_{\mathrm{f}} L_{\mathrm{m}} \sin \theta_{\mathrm{f}}+\mathrm{j}\left(1+\cos \theta_{\mathrm{f}}\right) \omega_{\mathrm{f}} L_{\mathrm{m}}
\end{aligned}
$$

where: $\omega_{\mathrm{f}}-$ the angular frequency of the inter-harmonics

In Fig. $4, \theta_{\mathrm{f}} \in\left(0^{\circ}, 180^{\circ}\right)$, so $\sin \theta_{\mathrm{f}}>0$. The equivalent resistance of the series transformer is a negative value.

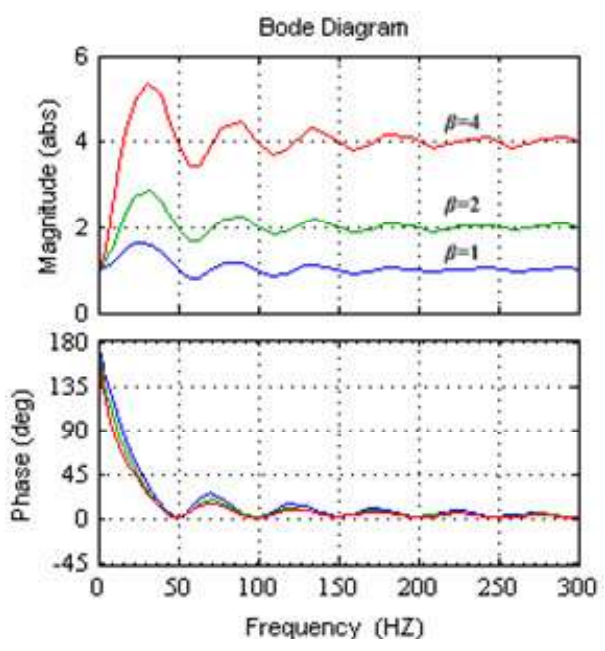

Fig. 4. Bode diagram of Eq. (5) with different $\beta$

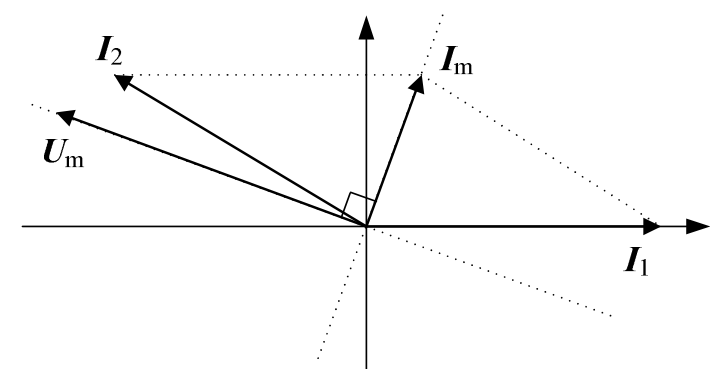

Fig. 5. Vector diagram of the series transformer 


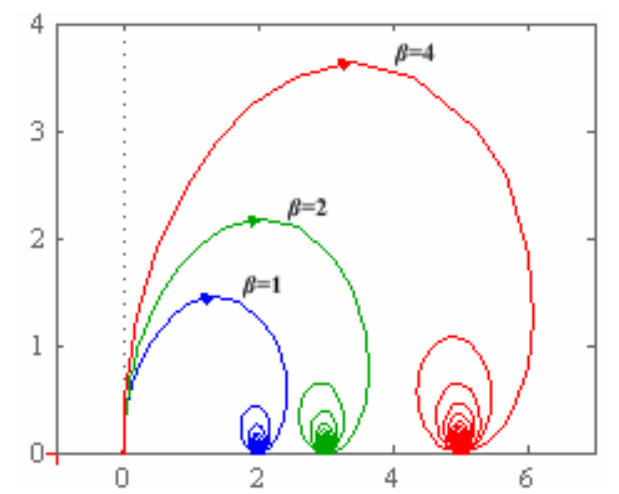

Fig. 6. Envelope curve of $\boldsymbol{I}_{\mathrm{m}}$ with different $\beta$

According to Fig. 5, $U_{\mathrm{m}}$ lies in the second quadrant, thus the real part of $\boldsymbol{U}_{\mathrm{m}}$ is less than 0 . That is the reason why the equivalent resistance of the series transformer is a negative value. And $\boldsymbol{I}_{\mathrm{m}}$ lie in the first quadrant is the reason why $\boldsymbol{U} \mathrm{m}$ lies in the second quadrant.

The amplitude and phase of $\boldsymbol{I}_{2}$ can be known according to Fig. 4, so the amplitude and phase of $\boldsymbol{I}_{\mathrm{m}}$ with different frequency inter-harmonics can be drawn out. Fig. 6 shows the envelope curve of the vector $\boldsymbol{I}_{\mathrm{m}}$. The direction of arrow represents the frequency of inter-harmonics $f_{\mathrm{pq}}$ increase direction.

Assume $\boldsymbol{I}_{\mathrm{m}}=a+\mathrm{j} b, a>0, b>0$. Then observing from the primary winding of the series transformer, the equivalent impedance to the inter-harmonic is:

$$
\begin{aligned}
Z_{\mathrm{t}} & =\frac{\dot{U}_{\mathrm{m}}}{\dot{I}_{1}}=\frac{\dot{I}_{\mathrm{m}} \times \mathrm{j} \omega L_{\mathrm{m}}}{\dot{I}_{1}}=\frac{\mathrm{j} \dot{I}_{\mathrm{m}} \times \mathrm{j} \omega_{\mathrm{f}} L_{\mathrm{m}}(a+\mathrm{j} b)}{\dot{I}_{1}} \\
& =-b \omega_{\mathrm{f}} L_{\mathrm{m}}+\mathrm{j} a \omega_{\mathrm{f}} L_{\mathrm{m}}
\end{aligned}
$$

From Eq. (7), the equivalent resistance and inductance of the series transformer to inter-harmonics are:

$$
\left\{\begin{array}{l}
R_{\mathrm{t}}=-b \omega_{\mathrm{f}} L_{\mathrm{m}} \\
L_{\mathrm{t}}=a L_{\mathrm{m}}
\end{array}\right.
$$

Eqs. (7) and (8) are equal to (6). From Eq. (8), the "equivalent negative resistance" of series transformer is proportional to the imaginary part of $\boldsymbol{I}_{\mathrm{m}}$. The "equivalent negative resistance" of the series transformer to the interharmonics will weaken the inter-harmonics stability of the APF system, discussed as follow.

The equivalent circuit of a single-phase APF system to both harmonics and inter-harmonics is shown as Fig. 7. $R_{\mathrm{t}}$ and $L_{\mathrm{t}}$ represent the series transformer, $L_{5}$ and $C_{5}$ represent the passive filter, $R_{\mathrm{s}}$ represent the system equivalent damping. $I_{\mathrm{h}}$ is the load harmonics and inter-harmonics current source; $U_{\text {sh }}$ is the power system harmonics and inter-harmonics voltage source. Since there is nearly no inter-harmonic in the system voltage and load current when the APF is not working, the inter-harmonic oscillation phenomenon is not generating by the system voltage

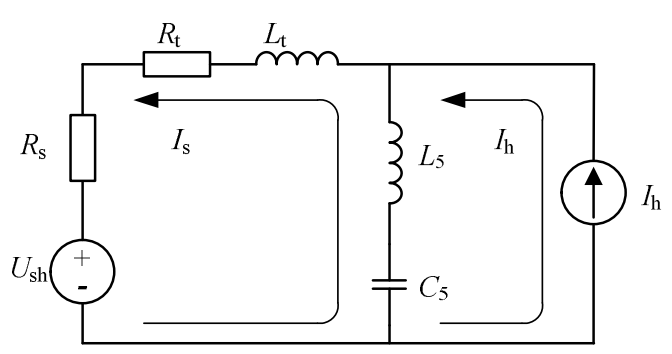

Fig. 7. Single phase equivalent circuit of SHAPF

source and load current source. To inter-harmonics, the source in Fig. 7 is 0 . That means $I_{\mathrm{h}}=0, U_{\mathrm{sh}}=0$. Assume parameters $L=L_{\mathrm{t}}+L_{5}, \quad R=R_{\mathrm{t}}+R_{\mathrm{s}}, \quad C=C_{5}$. Choosing the capacitor voltage $U_{\mathrm{C}}$ as state variable, the second-order differential equations of the circuit in Fig. 7 can be established:

$$
\frac{\mathrm{d}^{2} u_{\mathrm{C}}}{\mathrm{d} t^{2}}+\frac{L}{R} \cdot \frac{\mathrm{d} u_{\mathrm{C}}}{\mathrm{d} t}+\frac{1}{L C} u_{\mathrm{C}}=0
$$

The characteristic roots of the Eq. (9) are:

$$
\left\{\begin{array}{l}
\lambda_{1,2}=\frac{-R \pm \sqrt{\Delta}}{2 L} \\
\Delta=R^{2}-\frac{4 L}{C}
\end{array}\right.
$$

The general solution of Eq. (9) depends on the value of $\Delta$, as Table 1 shows. Each form of the general solution contains exponential function. If $\left|R_{\mathrm{t}}\right|>\left|R_{\mathrm{s}}\right|, R<0$. Thus when $\mathrm{t} \rightarrow \infty$, the solution of Eq. (9) $\rightarrow \infty$. The APF system is unstable in theory. The inter-harmonics stability of the APF system depends on the "equivalent negative resistance" of the series transformer. According to Fig. 6 and Eq. (8), APF system is unstable if $\beta$ is too large.

The premise of the above conclusion is that the inverter was considered as ideal controlled current source, and the

Table 1. General solution of $2^{\text {nd }}$ order differential equation.

\begin{tabular}{c|c|c}
\hline & Characteristic roots & General solution form \\
\hline \multirow{4}{*}{$\Delta>0$} & Unequal real root & \\
& $\left\{\begin{array}{l}\lambda_{1}=\frac{-R+\sqrt{\Delta}}{2 L} \\
\lambda_{2}=\frac{-R-\sqrt{\Delta}}{2 L}\end{array}\right.$ & $c_{1} e^{-\lambda_{1} t}+c_{2} e^{-\lambda_{2} t}$ \\
\hline \multirow{3}{*}{$\Delta=0$} & Equal real root & $\left(c_{1}+c_{2} t\right) e^{-\lambda t}$ \\
\hline$<0$ & $\left\{\begin{array}{l}\lambda=\frac{-R}{2 L} \\
\sqrt{\Delta}= \pm i \delta \\
\lambda_{1}=\frac{-R}{2 L}+i \delta \\
\lambda_{2}=\frac{-R}{2 L}-i \delta\end{array}\right.$ & $e^{\frac{-R t}{2 L}}\left(c_{1} \sin \delta t+c_{2} \cos \delta t\right)$ \\
\hline
\end{tabular}


inverter output current is equal to the reference current $I_{\text {ref }}$ all the time. But in practice, limited by the capacity of the actual inverter, the inverter can not track the system current to infinity. According to Fig. 4, after APF working, when the system current is increasing because of the "equivalent negative resistance" of the series transformer, the magnetizing voltage $U_{\mathrm{m}}$ will also increasing. If the peak value of $U_{\mathrm{m}}$ is large than the DC voltage, the inverter is out of control. Hence, with the increase of the system current, the inverter output current is not equal to the reference current. The actual inverter can not be considered as ideal controlled current source. The principle of the "equivalent negative resistance" of the series transformer is broken. So in the experiment, the system current will not increase to the infinity, but inter-harmonics oscillation occurs.

\subsection{Resolution method}

According to the above analysis, the essence of the interharmonic oscillation in the APF system based on MFC is that the APF system is unstable to the inter-harmonics. In order to solve this problem, the principle of the "equivalent negative resistance" of the series transformer must be weakening.

\subsubsection{Only compensate fundamental current}

The inverter output current contains no harmonic current when $\beta$ is equal to 0 . This is the traditional series hybrid APF based on the fundamental magnetic flux compensation. The stability of this APF has been proved by many literatures such as $[7-8,11]$.

Compared with the passive power filter, the major defect of active power filter is the high cost. New topologies and control strategies which can reduce the capacity of the inverter have been became the research hotspot of APF. In this series hybrid APF based on MFC, the harmonic component of the system current is very little after filtering. Adding harmonic current reference will not increase the capacity of the inverter but improve the filtering effect. So the harmonic current reference is significative.

\subsubsection{Voltage feed-forward control}

According to Fig. 5 and Fig. 6, the reason why the series establish "equivalent negative resistance" is that the imaginary part of $\boldsymbol{I}_{\mathrm{m}}$ is a positive value. If can reduce the imaginary part of $\boldsymbol{I}_{\mathrm{m}}$, make $R_{\mathrm{t}}$ in Eq. (8) less than the total system damping, the system will be stable to the interharmonics. In order to reduce the imaginary part of $\boldsymbol{I}_{\mathrm{m}}$, voltage feed-forward was proposed in this manuscript.

In this voltage feed-forward method, an additional reference current component $\boldsymbol{I}_{2}{ }^{\prime}$ is adding to the current reference. $\boldsymbol{I}_{2}{ }^{\prime}$ is proportional to $\boldsymbol{U}_{\mathrm{m}}$ and the proportion coefficient is a negative value. Since the leakage inductance can be ignored, $\boldsymbol{U}_{\mathrm{m}}$ is equal to the voltage of the

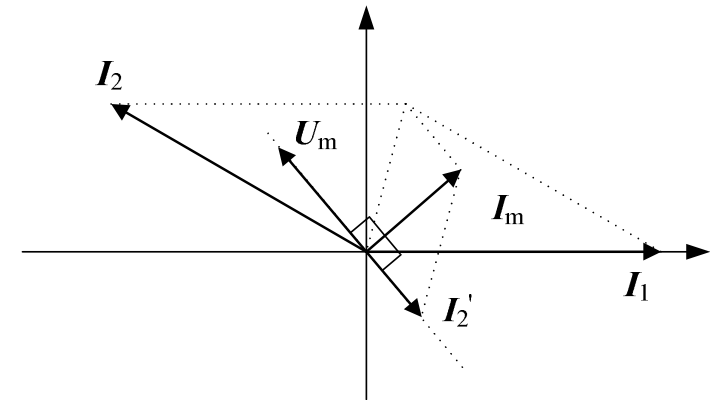

Fig. 8. Vector diagram of the series transformer while adding voltage feed-forward method

series transformer primary winding. The vector diagram of series transformer is shown as Fig. 8. $\boldsymbol{I}_{2}$ is the reference current obtained by Eq. (8). $\boldsymbol{I}_{2}^{\prime}$ is the voltage feed-forward control reference current. The total reference current is the sum of $\boldsymbol{I}_{2}$ and $\boldsymbol{I}_{2}{ }^{\prime}$. Compared with Fig. 5 , it is very clear that the imaginary part of $\boldsymbol{I}_{\mathrm{m}}$ in Fig. 8 is less. As a result, the inter-harmonics stability of APF system has been enhanced. The inter-harmonics oscillation will be eliminated. Beside $\boldsymbol{I}_{2}{ }^{\prime}$, there is not any other change of the series hybrid active power filter.

\section{Simulation and Experiments Verification}

\subsection{Simulation result}

The simulation circuit is shown as Fig. 9. The circuit parameters are equal to the experiment circuit in our laboratory. The mutual inductance between the primary winding and the secondary winding of the series transformer is $15 \mathrm{mH}$; the turns ratio of the series transformer is $1 / 1$; the leakage inductance of the primary winding is $0.5 \mathrm{mH}$; the passive power filter consists of 5 th turned LC filter, $L_{5}=2.13 \mathrm{mH}, C_{5}=68 \mathrm{uF}$, the capacitor is $\Delta$ connected. The number of $5^{\text {th }}$ turned passive filter is 3 (In Fig. 9, only 2 LC passive filter is plot out). The output inductance of each inverter is $1 \mathrm{mH}$. The load of the silicon controlled rectifier is $2.8 \Omega$ and $10 \mathrm{mH}$. In addition, a $0.5 \Omega$ resistance is series connected with each phase inverter in the simulation circuit to represent the total system damping.

Fig. 10 shows the simulation system current results with different conditions. The harmonics compensation coefficient $\beta$ is equal to 1 in every condition.

Using three ideal controlled current sources instead of the inverter in Fig. 9, system current is shown as Fig. 10(a). The system current shows that the APF system is unstable to the inter-harmonics. This simulation waveform validates the above analysis

Using three phase inverter in simulation, system current is shown as Fig. 10(b). The system current does not go to infinity, inter-harmonics oscillation occurred. The "beat" of the waveform in Fig. 10(b) is similar to the system current waveforms in Fig. 3. Of course the inter-harmonics 
oscillation in the experimental is more complex because of the errors in the actual system, such as the detection error and Analog-Digit convert error.

Fig. 10(c) shows the simulation system current when adding voltage feed-forward control. There is nearly no inter-harmonic oscillation in the system current. This simulation result verifies the validity of the voltage feedforward control.

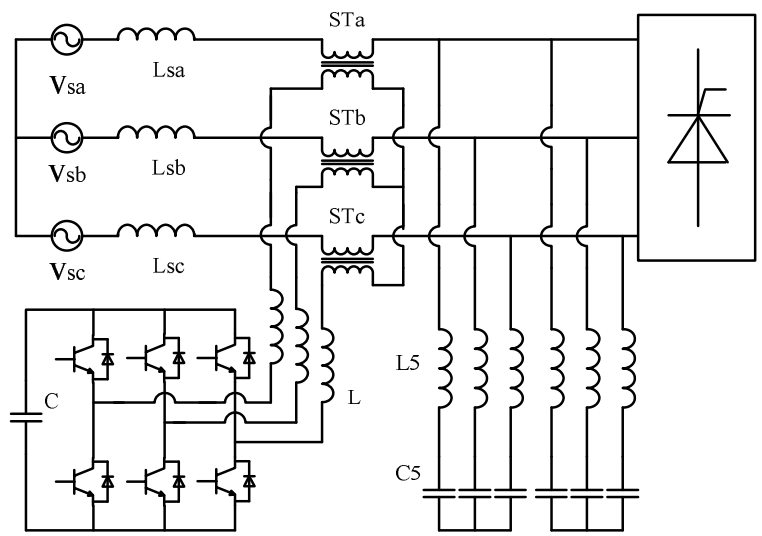

Fig. 9. Topology of series hybrid active power filter

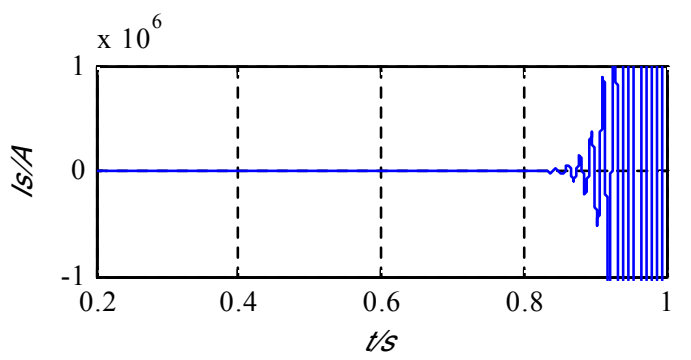

(a) system current using ideal controlled current source

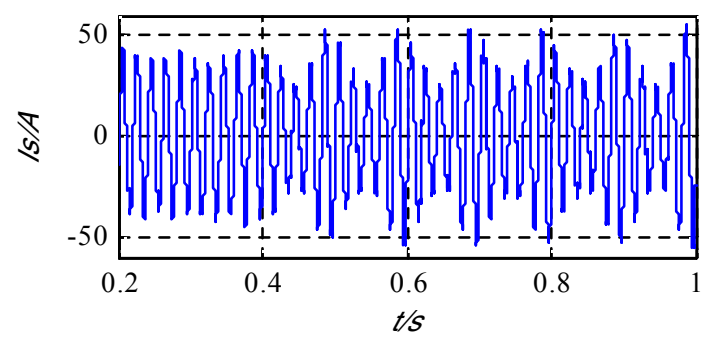

(b) system current using three-phase inverter

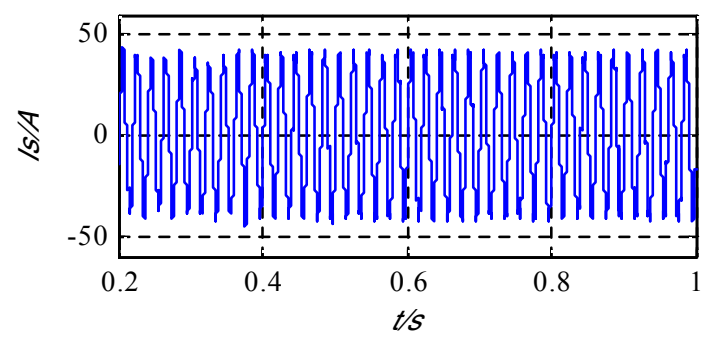

(c) system current when adopt voltage feed-forward control

Fig. 10. Simulation results
A prototype based on the system configuration shown in Fig. 9 has been built. The main circuit parameters are same to the simulation circuit. The capacitor at the DC side is $6800 \mu \mathrm{F} / 400 \mathrm{~V}$ electrolytic capacitor, 4 parallel and 2 series connection. The inverter uses IPM of PM300CLA120. The switching frequency is $12.8 \mathrm{kHz}$. The CPU chip of the APF system is the digital signal processor (DSP) TMS320F2812 manufactured by Texas Instruments (TI). Experimental waveforms are recorded by the TEK2000 digital oscilloscope.

Fig. 11(a) shows the system voltage and current waveforms while the 5th turned passive filter is under working. The rms value of the system current is $24.1 \mathrm{~A}$. The total harmonic distortion (THD) is $13.97 \%$.

Fig. 11(b) shows the inverter output current while $\alpha=1$, $\beta=1$. Compared with Fig. 11(a), the inverter output fundamental current is anti-phase with the system fundamental current, and the inverter output harmonic current is the same phase with the system harmonic current.

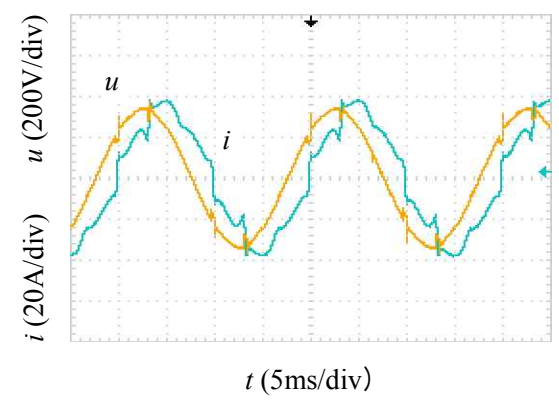

(a) System voltage and current while only PPF working

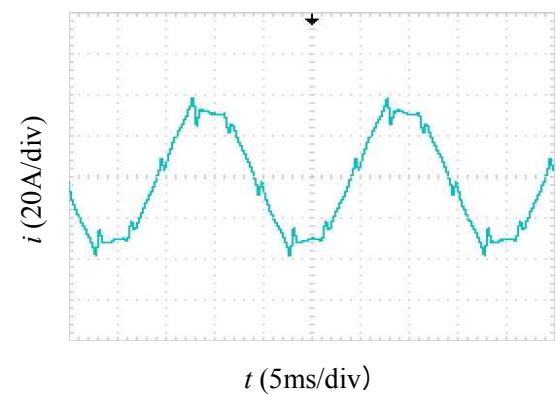

(b) The inverter output current before APF under working

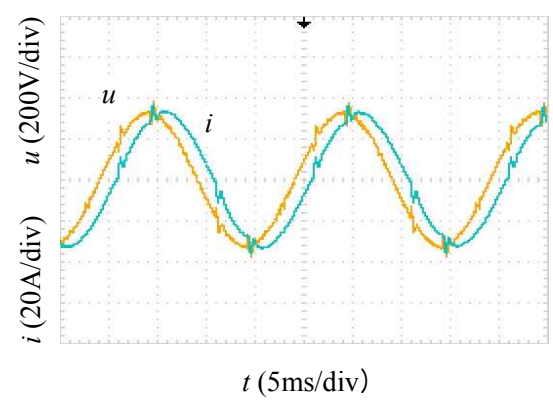

(c) System voltage and current while PPF and APF working

Fig. 11. Experiment results 
Fig. 11(c) shows the system voltage and current waveforms while the 5th turned passive filter and APF are all working. And voltage feed-forward control has been adding in the control block. The rms value of the system current is $23.1 \mathrm{~A}$. THD is $4.41 \%$. This experiment result verifies the validity and feasibility of the voltage feedforward control.

\section{Conclusion}

Research in this paper shows that the essence of interharmonics current oscillation in the SHAPF based on the magnetic flux compensation is that the APF is unstable to the inter-harmonics. If the "negative equivalent resistance" of the series transformer is larger than the system damping, APF is unstable to the inter-harmonics in theory. Limited by the capacity of the actual inverter, the inverter output current can not track the system current to infinity. Then the inter-harmonics oscillation happens. Though the conclusion of this paper is based on the period average algorithm LPF, the conclusion is also correct when adopt other LPF algorithms, such as Butterworth filter, and Bessel filter, et al.

This paper proposes the voltage feed-forward control to solve the inter-harmonics current oscillation problem. The validity and feasibility of the voltage feed-forward control are verified by both the simulation and experiment results.

\section{References}

[1] Chang G W, Hung-Lu Wang, Gen-Sheng Chuan and Shou-Yung, "Passive Harmonic Filter Planning in a Power System With Considering Probabilistic Constraints," IEEE Trans. Power Delivery, vol. 24, no.1, pp.208-218, Jan. 2009.

[2] EI-saadany E F, Salama M M A and Chikhani A Y, "Passive Filter Design for Harmonic reactive Power Compensation in Single-phase Circuits Supplying Nonlinear Loads," IEE Proceedings: Generation, Transmission and Distribution, vol. 147, no. 6, pp. 373-380, Nov. 2002.

[3] Wu Longhui, Zhuo Fang, Zhang Pengbo, Li Hongyu and Wang Zhaoan, "Study on the Influence of SupplyVoltage Fluctuation on Shunt Active Power Filter," IEEE Trans. Power Delivery, vol. 22, no. 3, pp. 17431749, July. 2007.

[4] Huayun Yang and Shiyan Ren, "A Practical SeriesShunt Hybrid Active Power Filter Based on Fundamental Magnetic Potential Self-Balance," IEEE Trans. Power Delivery, vol. 23, no. 4, pp. 2089-2096, Oct. 2008.

[5] Hirofumi Akagi and Pypta Kondo, "A Transformerless Hybrid Active Filter Using a Three-level
Pulsewidth Modulation (PWM) Converter for a Medium-Voltage Motor Drive," IEEE Trans. Power Electron, vol. 25, no. 6, pp. 1365-1374, June. 2010.

[6] Zhikang Shuai, An Luo, Shen John and XiaoWang, "Double closed-loop control method for injectiontype hybrid active power filter," IEEE Trans. Power Electron, vol. 26, no. 9, pp. 2393-2403, Sept. 2011.

[7] Dayi Li, Qiaofu Chen, Zhengchun Jia and Jianxing $\mathrm{Ke}$, "A novel active power filter with fundamental magnetic flux compensation," IEEE Trans. Power Delivery, vol. 19, no. 2, pp. 799-805, Apr. 2004.

[8] Dayi Li, Qiaofu Chen, Zhengchun Jia and Chang zheng Zhang, "A High-Power Active Filtering System With Fundamental Magnetic Flux Compensation," IEEE Trans. Power Delivery, vol. 21, no. 2, pp. 823830, Apr. 2006.

[9] Zhang Yu, Chen Qiaofu, Tian Jun, Li Xin and Li Jianhui, "Controllable Reactor Based on Transformer Winding Current Regulating," Proceedings of the CSEE, vol. 29, no. 18, pp. 113-118, June. 2009.

[10] Jun Tian, Qiaofu Chen, Lu Cheng and Changzheng Zhang, "A novel shunt hybrid active power filter based on magnetic flux compensation," International Journal of Electronics, vol. 96, no. 10, pp. 1071-1084, Oct. 2009.

[11] Bingruo Xie, Qiaofu Chen, Jun Tian, Lu Cheng and Jin Wang, "Analysis and improvements of the filtering characteristic of the series hybrid APF based on fundamental magnetic flux compensation," in Proceedings of IEEE ICEMS2007 Conference, Seoul, Korea, Oct 2007.

[12] Jiang Bin, Yan Gangfeng and Zhao Guangzhou, "Study on a detecting method for harmonic currents in single-phase circuit," Transactions of China Electrotechnical Society, vol. 15, no. 6, pp. 65-69, Dec. 2000.

[13] Tong Liqing, Qian Zhaoming and Peng Fangzheng, "Synchronous reference frame harmonic detection modeling and digital realization," Proceedings of the CSEE, vol. 29, no. 19, pp. 111-117, July. 2009.

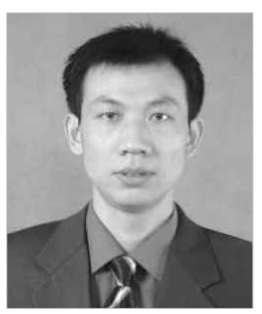

Zhang Jian-gong $\mathrm{He}$ was born in Hubei, China, in 1975. He received the B.S. degree in electrical engineering from Wuhan University of Hydraulic and Electric, Wuhan, China, in 1999, and the M.S. degree in electrical engineering from Huazhong University of Science and Technology (HUST), Wuhan, China, in 2006. Currently, He is with the China Electric Power Research Institute (CEPRI), Wuhan City, Hubei Province, China. His research interests include power system electromagnetic compatibility and the electromagnetic environment of power systems. 


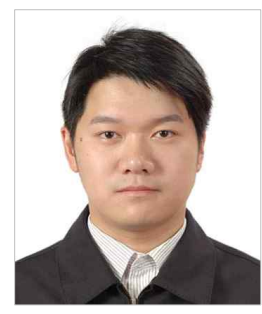

Liu Jian-ben He received the B.S. and $\mathrm{Ph} . \mathrm{D}$. degrees in electrical engineering from Huazhong University of Science and Technology (HUST), Wuhan, China, in 2007 and 2013, respectively. He is currently working as an engineer in the China Electric Power Research Institute (CEPRI), Wuhan City, Hubei Province, China. His research interests include power electronics, harmonic suppression and reactive power compensation technology.

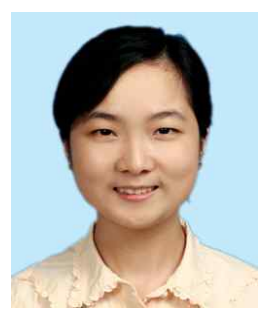

Dai Shao-jun She received the B.S. degree in electrical engineering from Huazhong University of Science and Technology (HUST), Wuhan, China, in 2009. She is currently working in Electric Power Economic and Technological Research Institute of State Grid Wuhan Power Supply Company, as an assistant engineer. Her working content includes power system planning, scientific and technical literatures translation.

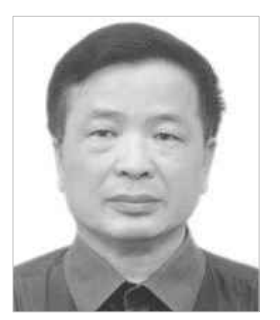

Chen Qiao-fu He received the B.S. and M.S. degrees from Huazhong University of Science and Technology (HUST), Wuhan, China, in 1968 and 1982, respectively. He is currently a professor of HUST. His research interests are flexible ac transmission systems (FACTS), harmonic suppression and active power filters, transformers, reactors, and fault current limiters. Mr. Chen is a member of the transformer subcommittee of the China Society of Electrical Engineering and is a member of the transformer standard of China.

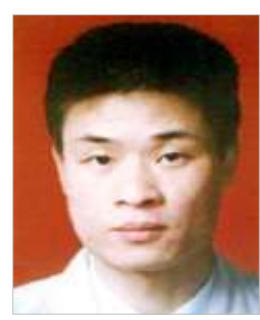

He Jun-jia He was born in Hunan, China, in 1968. He received the B.S. and Ph.D. degrees from Huazhong University of Science and Technology (HUST), Wuhan, China, in 1990 and 1995, respectively. He was a research student with Nagoya University, Nagoya, Japan, from October 1999 to September 2001. Since 2001, he has been a professor with HUST. His current research interests include high-power pulsed technology and high voltage engineering. 